\title{
RETROPERITONEAL LYMPH NODE RECURRENCE OF EPITHELIAL OVARIAN CANCER: PROGNOSTIC FACTORS AND TREATMENT OUTCOME
}

Levy T, MD, MHA; Migdan Z, MD, Peled O, MD, Ben Shem E, MD, Elyashiv O, MD

OBSTETRICS \&GYNECOLOGY WOLFSON MEDICAL CENTER

Division of Gynecologic Oncology, Department of Obstetrics and Gynecology, E. Wolfson Medical Center, Holon, Israel, Sackler Faculty of Medicine, Tel Aviv University, Israel

Aim: To compare the treatment outcome and survival of patients with recurrence in retroperitoneal lymph nodes versus women with intraperitoneal recurrence.

Methods: This retrospective cohort study included patients treated for ovarian, tubal and primary peritoneal cancer in Wolfson Medical Center during the years 2000-2015. We classified three groups according to the site of recurrence: intraperitoneal only, retroperitoneal lymph nodes only, and both. Response to treatment was assessed by the RECIST criteria. Progression free survival (PFS), postrecurrence survival (PRS) and overall survival (OS) were estimated with the Kaplan-Meier method and compared with Log-rank test. The association between clinical variables and survival was established by Cox proportional hazards model.

Results: Out of 135 patients in our cohort, 66 were diagnosed with intraperitoneal recurrence, 30 with retroperitoneal lymph node recurrence and 39 with combined recurrence. The clinical, pathological and surgical characteristics were similar among all groups, besides CA-125, which was significantly lower in the retroperitoneal recurrence group at diagnosis, end of treatment and recurrence. The median follow up period was 45.8 months. OS and PRS were significantly higher in the retroperitoneal recurrence group vs. the intraperitoneal and combined recurrence groups. (OS - 93.07 vs. 47.9 and 41.7 months, respectively, $p=0.000$, PRS -68.57 vs. 29.67 and 19.7 months, respectively, $\mathrm{p}=0.000)$. In multivariate analysis, retroperitoneal recurrence was found to be an independent prognostic factor for survival.

Conclusions: The site of recurrence has significant prognostic value regarding PRS and OS. Patients with retroperitoneal lymph node recurrence only, have a favorable prognosis with estimated survival longer than 5 years.

\begin{tabular}{|c|c|c|c|c|c|}
\hline Variables & $\begin{array}{l}\text { Intra- } \\
\text { peritoneal } \\
\text { recurrence } \\
(\mathrm{N}=66)\end{array}$ & $\begin{array}{l}\text { Retro-peritoneal } \\
\text { recurrence } \\
(\mathrm{N}=30)\end{array}$ & $\begin{array}{l}\text { Combined } \\
\text { recurrence } \\
\text { (N=39) }\end{array}$ & $\begin{array}{l}\text { Total } \\
(\mathrm{N}=135)\end{array}$ & $\begin{array}{l}P \text { - } \\
\text { value }\end{array}$ \\
\hline $\begin{array}{l}\text { Age at diagnosis } \\
\text { (median,years) }\end{array}$ & 65.44 & 64.99 & 64.75 & 65.04 & NS \\
\hline $\begin{array}{l}\text { Stage } \\
\text { I } \\
\text { II } \\
\text { III } \\
\text { IV }\end{array}$ & $\begin{array}{l}2(3 \%) \\
2(3 \%) \\
57(86.4 \%) \\
5(7.6 \%)\end{array}$ & $\begin{array}{l}2(6.7 \%) \\
0(0 \%) \\
26(86.6 \%) \\
2(6.7 \%)\end{array}$ & $\begin{array}{l}1(2.6 \%) \\
2(5.1 \%) \\
28(71.8 \%) \\
8(20.5 \%)\end{array}$ & $\begin{array}{l}5(3.7 \%) \\
4(3 \%) \\
111(82.2 \%) \\
15(11.1 \%)\end{array}$ & NS \\
\hline $\begin{array}{l}\text { Response rate to } \\
\text { first chemo } \\
\text { Complete } \\
\text { Partial } \\
\text { Progressive } \\
\text { Stable disease }\end{array}$ & $\begin{array}{l}49(74.2 \%) \\
10(15.2 \%) \\
4(6.1 \%) \\
3(4.5 \%)\end{array}$ & $\begin{array}{l}25(83.3 \%) \\
4(13.3 \%) \\
1(3.3 \%) \\
0\end{array}$ & $\begin{array}{l}31(79.5 \%) \\
5(12.8 \%) \\
1(2.6 \%) \\
2(5.1 \%)\end{array}$ & $\begin{array}{l}104(77.8 \%) \\
19(14.1 \%) \\
6(4.4 \%) \\
5(3.7 \%)\end{array}$ & NS \\
\hline $\begin{array}{l}\text { Platinum } \\
\text { Sensitive } \\
\text { Resistant } \\
\text { Refractory }\end{array}$ & $\begin{array}{l}39(59.1 \%) \\
20(30.3 \%) \\
7(10.6 \%)\end{array}$ & $\begin{array}{l}22(73.3 \%) \\
7(23.3 \%) \\
1(3.3 \%)\end{array}$ & $\begin{array}{l}24(61.5 \%) \\
12(30.8 \%) \\
3(7.7 \%)\end{array}$ & $\begin{array}{l}85(63 \%) \\
39(28.9 \%) \\
11(8.1 \%)\end{array}$ & NS \\
\hline $\begin{array}{l}\text { Mean CA-125 } \\
\text { At diagnosis } \\
\text { Mid treatment } \\
\text { End of treatment } \\
\text { At recurrence }\end{array}$ & $\begin{array}{l}2003.80 \\
103.63 \\
29.59 \\
236.5\end{array}$ & $\begin{array}{l}722.41 \\
122.03 \\
11.97 \\
56.5\end{array}$ & $\begin{array}{l}1706.57 \\
452.58 \\
87.94 \\
631.8\end{array}$ & $\begin{array}{l}1636.18 \\
205.95 \\
41.78 \\
306.9\end{array}$ & $\begin{array}{l}P=0.002 \\
N S \\
P=0.018 \\
P=0.03\end{array}$ \\
\hline $\begin{array}{l}\text { Type of surgery } \\
\text { Primary } \\
\text { Interval } \\
\text { None }\end{array}$ & $\begin{array}{l}36(54.5 \%) \\
26(39.4 \%) \\
4(6.1 \%)\end{array}$ & $\begin{array}{l}17(56.7 \%) \\
13(43.3 \%) \\
0(0 \%)\end{array}$ & $\begin{array}{l}17(43.6 \%) \\
20(51.3 \%) \\
2(5.1 \%)\end{array}$ & $\begin{array}{l}70(51.9 \%) \\
59(43.7 \%) \\
6(4.4 \%)\end{array}$ & NS \\
\hline $\begin{array}{l}\text { Debulking } \\
0 \mathrm{~cm} \\
0-1 \mathrm{~cm} \\
>1 \mathrm{~cm} \\
\quad \text { Not operated }\end{array}$ & $\begin{array}{l}37(46.1 \%) \\
15(22.7 \%) \\
10(15.1 \%) \\
4(6.1 \%)\end{array}$ & $\begin{array}{l}18(60 \%) \\
\%) 23.3(7 \\
5(16.7 \%) \\
0(0 \%)\end{array}$ & $\begin{array}{l}17(43.6 \%) \\
15(38.5 \%) \\
5(12.8 \%) \\
2(5.1 \%)\end{array}$ & $\begin{array}{l}72(53.3 \%) \\
\%) 27.4(73 \\
20(14.8 \%) \\
6(4.4 \%)\end{array}$ & NS \\
\hline $\begin{array}{l}\text { Pelvic lymph nodes } \\
\text { dissection } \\
\text { Yes } \\
\text { No } \\
\text { Not operated }\end{array}$ & $\begin{array}{l}24(36.4 \%) \\
38(57.6 \%) \\
\%) 04(6 .\end{array}$ & $\begin{array}{l}17(56.7 \%) \\
13(43.3 \%) \\
0(0 \%)\end{array}$ & $\begin{array}{l}22(56.4 \%) \\
15(38.5 \%) \\
2(5.1 \%)\end{array}$ & $\begin{array}{l}63(46.7 \%) \\
66(48.9 \%) \\
6(4.4 \%)\end{array}$ & NS \\
\hline $\begin{array}{l}\text { Para-Aortic lymph } \\
\text { nodes dissection } \\
\text { Yes } \\
\text { No } \\
\text { Not operated }\end{array}$ & $\begin{array}{l}14(21.2 \%) \\
48(72.7 \%) \\
4(6.1 \%)\end{array}$ & $\begin{array}{l}12(40 \%) \\
18(60 \%) \\
0(0 \%)\end{array}$ & $\begin{array}{l}9(23.1 \%) \\
28(71.8 \%) \\
2(5.1 \%)\end{array}$ & $\begin{array}{l}35(25.9 \%) \\
94(69.6 \%) \\
6(4.4 \%)\end{array}$ & NS \\
\hline
\end{tabular}
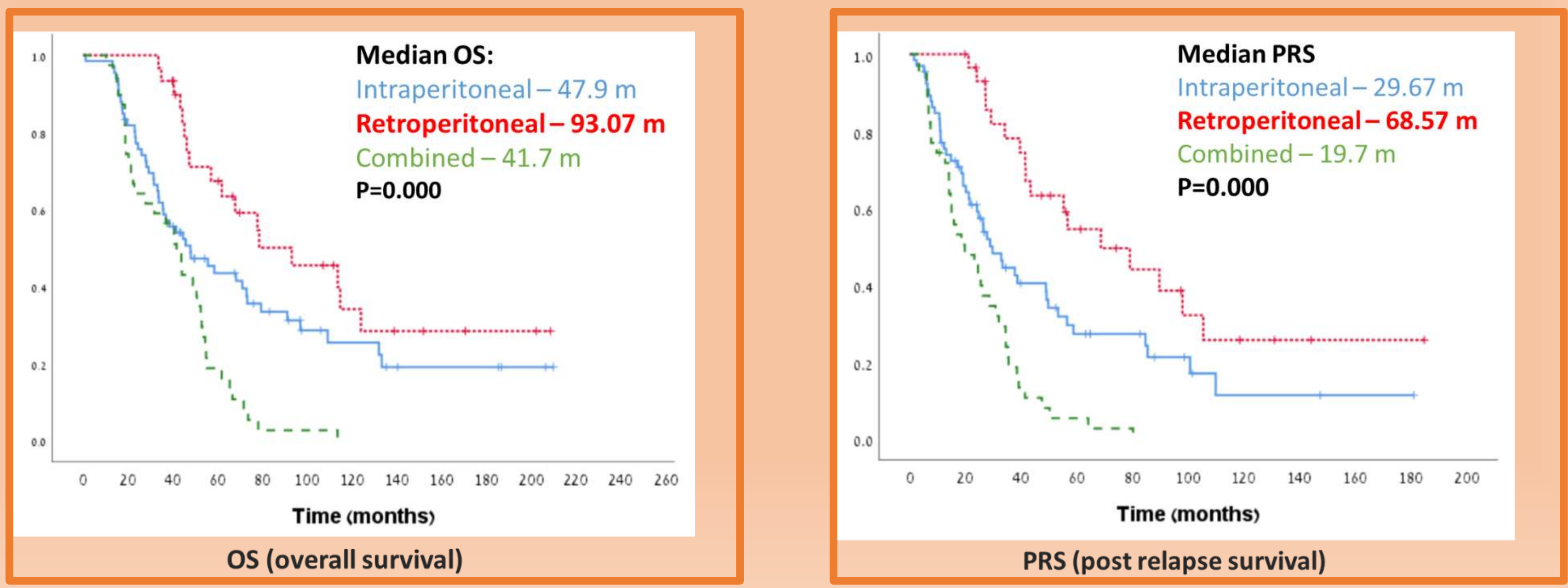